\title{
Results and Survival at Mid-term Follow-up of a Series of 15 High Tibial Lateral Closing Wedge Osteotomies in the Treatment of Unicompartimental Medial Osteoarthritis
}

\author{
Souna B Seyni*, Amadou S, Farikou I, Zirbine AS, Abdoul Wahab M and Moumouni H
}

Service d'orthopédie-traumatologie CHU Lamordé, Niamey, Niger

\begin{abstract}
The osteotomy of the proximal tibia remains widely practiced by orthopedic surgeons in treating unicompartmental knee osteoarthritis. A retrospective study was carried out on a group of 15 patients who were operated in our Orthopedics and Traumatology Department over a period of six years. Patients underwent valgus closing wedge osteotomy of the proximal tibia in the treatment of femoro-tibial medial knee osteoarthritis. The purpose of this study was to evaluate the results and the medium-term survival of this series of 15 osteotomies. We recorded $13.3 \%$ of superficial infections of the surgical wound and $6.6 \%$ nonunion. Despite radiological signs of deterioration of the knees, the percentage excellent results in more than 10 years of follow up on the entire series were $66.6 \%$.

This study demonstrated that, well performed, wedge closing wedge osteotomy of the proximal tibia significantly delays the age of the prosthetic knee replacement or even prevents the patient from a total knee replacement.
\end{abstract}

Keywords: Knee medial osteoarthritis; High tibial osteotomy; Closing wedge

\section{Introduction}

The osteotomy of the proximal tibia remains widely practiced by orthopedic surgeons in the surgical management of the osteoarthritis of the medial compartment of the knee

The three common techniques are: the tibia wedge external closing osteotomy, the tibia internal opening osteotomy and the tibia curvilinear or dome osteotomy [1]. The high tibial osteotomy of valgisation (closing wedge), was described for the first time in 1961 by Jackson and Waugh [2], the technique was popularized by Coventry [3] in 1965.

The contribution of these tibia osteotomies is to correct the mechanical axis to tip the weight of the medial compartment defect, toward the lateral compartment less involved by osteoarthritis. In the case of the closing wedge osteotomy, the site is located at the top of the anterior tibia tuberosity and it will be necessary to remove a bone corner sufficiently enough to obtain the necessary valgus.

This lateralization of the mechanical axis of the member will contribute to improve the function of the member by the disappearance of the pain. A retrospective study was carried out for a group of 15 patients who were operated in our Trauma and Orthopedic Service from January 2003 to December 2008. These patients underwent valgus osteotomy in the treatment of medial tibiofemoral knee osteoarthritis. The purpose of this study is to assess the results and the medium-term survival of this series of osteotomies.

\section{Patients and Method}

The study involved 15 patients of both sexes who underwent high tibial valgus closing wedge osteotomy for medial femorotibial knee osteoarthritis at our Trauma and Orthopedic Service. It was a retrospective study which was carried out over a period of 6 years from 1st January 2003 to 31 December 2008. The average age of our patients was 58.6 years (36 years to 80 years). There were 13 women (86.6\%) and 2 men (13.4\%). Were included in this study, all patients who underwent valgus osteotomy for medial femoro-tibial osteoarthritis whose symptoms are not relieved by medical treatment alone, all patients whose medical record contains the clinical and radiological information most complete possible with a minimum of 5 years of follow up. Were not included in this study, patients with knee osteoarthritis due to genu varum treated by opening wedge osteotomy or dome osteotomy. Patients operated outside this period of study were not included.

At last check up, all patients underwent enquiry, clinical and diagnostic testing including regarding age, sex, occupation, looking for complications related to the surgical procedure performed as a fracture, a thrombo-embolic complication, an infection, vascular and nerve injury, recurrence of the varus, the occurrence of nonunion, functional gain brought by the surgery, the persistence or disappearance of knee pain.

The radiological assessment included anterposterior view in monopodal support and and lateral view at $30^{\circ}$ of flexion. This allowed us to evaluate the preoperative and postoperative valgus deformity. The discovered knee osteoarthritis are classified according to the different stages described by Ahlbäck.

The Lequesne index (lying face-down) was measured (preoperatively and after one year interval): this is based on the pain or functional discomfort, the perimeter of maximum walking, the other difficulties of daily life activities. The total score varies from 0 to 26.The expected result of the osteotomies of tibial valgisation was to obtain a maximum tibia valgus of $3^{\circ}$ to $6^{\circ}$ according to Hernigou [4].

The osteotomies were fixed either by Blount staples and long leg cast, or epiphyseal $\mathrm{T}$ shape plate.

*Corresponding author: Souna B Seyni, service d'orthopédie-traumatologie CHU, bLamorde, Niamey, Niger, Tel: 00227938001 42; E-mail: badioseyni@yahoo.fr

Received October 20, 2014; Accepted January 20, 2015; Published January 24, 2015

Citation: Seyni SB, Amadou S, Farikou I, Zirbine AS, Wahab AM, et al. (2015) Results and Survival at Mid-term Follow-up of a Series of 15 High Tibial Lateral Closing Wedge Osteotomies in the Treatment of Unicompartimental Medial Osteoarthritis. J Trauma Treat 4: 234. doi:10.4172/2167-1222.1000234

Copyright: ( 2015 Seyni SB, et al. This is an open-access article distributed under the terms of the Creative Commons Attribution License, which permits unrestricted use, distribution, and reproduction in any medium, provided the original author and source are credited. 
Citation: Seyni SB, Amadou S, Farikou I, Zirbine AS, Wahab AM, et al. (2015) Results and Survival at Mid-term Follow-up of a Series of 15 High Tibial Lateral Closing Wedge Osteotomies in the Treatment of Unicompartimental Medial Osteoarthritis. J Trauma Treat 4: 234. doi:10.4172/2167-1222.1000234

\section{Results}

In preoperative, the average body mass index (BMI) of our patients was $28.6 \mathrm{~kg} / \mathrm{sq}$. m with extremes ranging from $22.1 \mathrm{Kg} / \mathrm{sq}$. $\mathrm{m}$ to 38.8 $\mathrm{kg} / \mathrm{sq}$. m. Fifty percent of our patients had a BMI $\geq 30 \mathrm{~kg} / \mathrm{sq}$. m. In preoperative also, the average range of motion of passive flexion was $100^{\circ}$ with the extremes ranging from $60^{\circ}$ to $140^{\circ}$. The average range of motion of active flexion was $98^{\circ}$ with the extremes ranging from $50^{\circ}$ to $140^{\circ}$. No case of flexum has been notified. The average of Lequesne index (lying face-down) was 19 with the extremes ranging from 14 to 24. Among this series of 15 patients, 7 patients were affected by stage 2 knee osteoarthritis and 5 patients had stage 3 knee osteoarthritis and 3 patients had a stage 4 knee osteoarthritis according to Ahlbäck classification (Figure 1). The mean of preoperative value of varus measured on the orthroengenogram anteroposterior view was $8.9^{\circ}$ (extremes from $6^{\circ}$ to $23^{\circ}$ ).

In postoperative, we reported:

$11(73.3 \%)$ cases of fractures (hinge and joint shear) during the realization of the osteotomy, including 9 cases of fractures of the opposed cortical hinge and 2 cases of joint shear.

$2(13.3 \%)$ cases of superficial surgical site infections treated by intensive local dressing and by oral route antibiotic therapy, without requiring the removal of material of osteosynthesis), No case of thromboembolic complication was observed.

\section{After one year of postoperative follow up}

The preoperative average range of motion of passive flexion was 110 degrees with the extremes ranging from 90 to 140 degrees, that of active flexion was 105 degrees with the extremes ranging from 90 to 130 degrees. 1 (6.6\%) case of pseudarthrosis, associated with a peroneal nerve palsy ( at the time of the surgical intervention) had occurred in a 70 years old patient with a $\mathrm{BMI}=33.5 \mathrm{~kg} / \mathrm{m}^{2}$, and who presented a stage 4 knee osteoarthritis with a hyphen osteotomy site located below the anterior tibial tuberosity. The normocorrection was obtained in 9 (60\%) patients, the hypercorrection was obtained in 5 patients is $33.3 \%$ and the hypocorrection was occurred in one patient (6.7\%).

\section{After 5 year of postoperative follow up}

The patient who suffered from the pseudarthrosis, has been cured of the latter thanks to a recovery by epiphyseal $\mathrm{T}$ shape plate with addition of bone substitute, but without the recovery of its nerve paralysis. On clinical plan, the other 14 patients were doing well with disappearance of the knee pain, and the normal mobility of the knee, without the recurrence of the varus and without limitation of the perimeter of walking. In sum the knee was "forgotten" by each of these patients. Thus all patients who were suffering from bilateral knee osteoarthritis, planned to do operate the contralateral knee. On radiological plan: the height of the medial femoro-tibial interline was retained without sign of radiological degradation. As well we can lay claim after 5 years of follow up $93.3 \%$ of excellent results.

\section{Between the tenth and twelfth year period after surgery}

Two patients had died, and three others were lost of sight. The other 10 patients who were examined, do not still complain of their knee on functional plan. Whereas on radiological plan, there is a degradation of the femoro-tibial medial interline by the pinching and the presence of osteophytes. Despite the signs of radiological degradation, the patients did not complain of any of their knees on functional plan with $66.6 \%$ of excellent results to more than 10 years of follow up on the whole series.

\section{Discussion}

\section{Epidemiological profile}

The average age of our patients was 58.6 years (36 to 80 years). They were 13 women $(86.6 \%)$ and 2 men $(13.4 \%)$. This average age is favorably comparable to that reported by Bouharras et al. [5] which was 58 years. The osteotomy of the proximal tibia due to knee osteoarthritis due to genu varum is thus indicated in younger subjects that delaying the indication of knee prosthesis.

The female sex predominates in our series with $26(86.6 \%)$ cases. These results are close to those reported by Dunoyer et al. [6]. who find $86 \%$ of women in a series of 288 knees, and Eti et al. [7] with $57 \%$ of female subjects over a total of 32 cases. Rahmi et al. [8] reported $80.24 \%$ of female subjects on 240 records. Fifty percent of our patients had a $\mathrm{BMI} \geq 30 \mathrm{~kg} / \mathrm{sq}$. $\mathrm{m}$. This predominance of female subjects is explained by the link between knee osteoarthritis due to genu varum and obesity among women in our country.

The majority of our patients had consulted at a late stage (8 patients over 15) and the osteotomy was performed on advanced knee osteoarthritis (stage 3 and 4 of Ahlbäck. Thus our functional results are presumed to be less good.

\section{Complications}

\section{Infection}

The infection rate caused by closing wedge osteotomy of the proximal tibia varies from $0.8 \%$ to $10 \%$ [9-12]. With $2(13.3 \%)$ cases of superficial infections of surgical site, our results seem less good than those of the authors cited above.

The tropical environment is undoubtedly a factor favoring the onset of infection. In contrast the superficial nature of these infections is reflected both in our study that reported by Naudie et al. [9]. These infections have been successfully treated by oral route antibiotic therapy and local intensive care, without the slightest threat to the hardware of osteosynthesis.

\section{Thromboembolic events}

According to some authors $[9,13]$ the incidence of venous thrombosis varies from $2 \%$ to $5 \%$ in the tibial wedge closing external

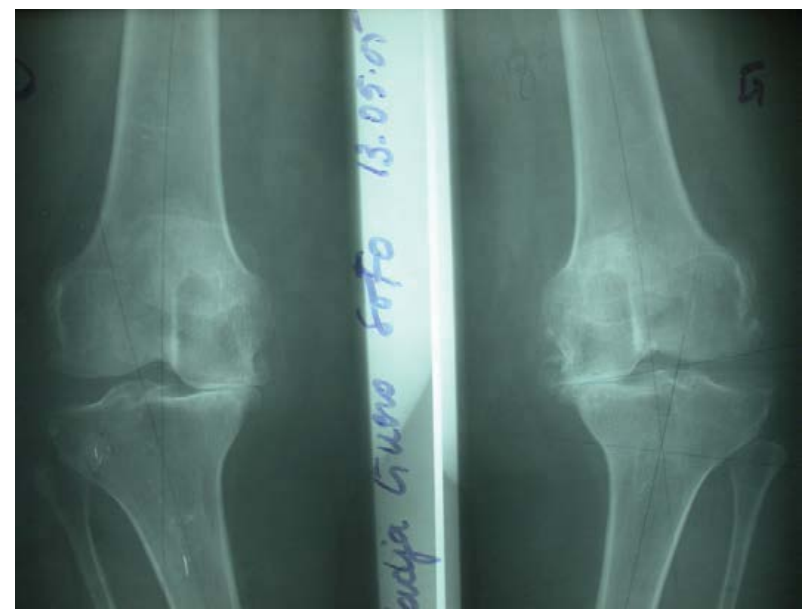

Figure 1: Gonarthrose stage 4 of Ahalback of both knees before the surgical correction. 
Citation: Seyni SB, Amadou S, Farikou I, Zirbine AS, Wahab AM, et al. (2015) Results and Survival at Mid-term Follow-up of a Series of 15 High Tibial Lateral Closing Wedge Osteotomies in the Treatment of Unicompartimental Medial Osteoarthritis. J Trauma Treat 4: 234. doi:10.4172/2167-1222.1000234

osteotomy. In our series, we did not observe any case of thromboembolic event. Is it a lack genuine or a difficulty to diagnose this complication our patients? We have not performed postoperative venography in our patients, our diagnosis being mainly based on clinical signs. Turner et al. [14] in performing a postoperative venography in their patients, have demonstrated the existence of a deep vein thrombosis in $41 \%$ of patients.

But among the vast majority of patients, venous thrombosis is localized in the distal portion of the calf with a low risk of migrating to the top. Even in the case of migration of the clot upwards, only $15 \%$ of patients will present a full clinical pathognomonic feature of this accident $[13,15]$. Thus the systematic use of anticoagulants in patients after tibial osteotomy closure is it justified?

\section{Fracture}

The osteotomy can be considered as a controlled fracture. However, during the technical realization of this operation, one of the two following complications may occur: the fracture of the hinge (medial cortex) and joint shear. We reported $11(73.3 \%)$ cases of fractures (hinge and joint shear) during the realization of the osteotomy (Figures 2 and 3 ). These fractures did not need any additional osteosynthesis, but a long leg cast immobilization for 1 months was sufficient to allow the consolidation (Figure 3).

The joint shear occurred in two patients operated at the beginning of our experience. Bone subtracted at the external wedge osteotomy remains bound leaving a thickened hinge (Figure 4) requiring significant force to be applied to the inner cortex for the valgus. It was during this operation that occurs articular shear

Regarding the fracture of the hinge, it was in our opinion the result of overcorrection because we have sought a valgus at least $6^{\circ}$ every time.

Thus for Kessler et al. and Mathews et al. [16,17], this fracture of the hinge is not totally preventable. This complication is even inevitable in case of valgus greater than $7^{\circ}$ to $8^{\circ}$. The fracture of the hinge and the joint shear caused no cases of nonunion or recurrence of the varus in our series.

\section{Vascular and nerve lesions}

We did not observe any case of vascular impairment. However we recorded 1(6.6\%) cases of infringement of the spiral cubital nerve. The incidence of occurrence of peroneal nerve paralysis during the realization of the tibial external wedge closing osteotomy closing varies between 3.3 per cent to 11.9 per cent. The EMG demonstrated that achieving this nerve has a frequency greater than $27 \%[18,19]$. In our study, this reach the nerve is iatrogenic by a track first located virtually in the intertibiofibular depression. According to Aydogdu et al and Woutton et al. $[18,20]$, the frequent cause of injury to the nerve is iatrogenic, in report especially with the topographic situation of the osteotomy of the fibula.

\section{The pseudarthrosis}

One of the advantages of the wedge closing osteotomy is its low rate of pseudarthrosis; because of the good lens compression afforded by the external closure, this rate is between $1 \%$ to $5 \%[2,12,19]$. We recorded $1(6.6 \%)$ case of pseudarthrosis in a patient age of 70 years (at the time of the osteotomy), suffering from an infringement of the iatrogenic spiral cubital nerve and whose the hyphen osteotomy stood below the anterior tibial tuberosity.

We suspect the influence of the age and especially the situation of the hyphen osteotomy in the occurrence of this pseudarthrosis. From a point of surgical view, it is considered that there is a relationship between the situation from the point of osteotomy (below the anterior tibial tuberosity) and the occurrence of delay of consolidation or pseudarthrosis [21]. Furthermore, we have not noticed a difference in function of the means of securing (Blount staples and $\mathrm{T}$ shape plate), with regard to the occurrence of the pseudarthrosis.

\section{Functional Results}

The external closing wedge tibial osteotomy as treatment of the medial femoro-tibial osteoarthritis has for main effect to defer $70 \%$ of the weight of the medial interline to the femoro-tibial interline side which is more healthy, thus slowing down the arthritic degradation. According to Amendola and Bonasia [22], this technique gives good results in young subjects, without ligamentous laxity, nor flexum. What is favorably comparable to our results, in particular with an average age of the series which is 58.6 years ( 36 to 80 years old).

To 5 years period of follow up, on the clinical plan, the 14 patients on our 15 patients have "forgoten" their knee. On the radiological plan, the height of the femoro-tibial medial interline was retained without sign of arthritic degradation.

Thus we recorded $93.3 \%$ of excellent results. This result is comparable to that reported by Spahn et al. [23], which is $91 \%$ after 5 to 8 years. This confirms that, if conducted on good ground, external wedge closing tibial osteotomy provides an excellent medium-term results in the treatment of isolated medial femoro-tibial osteoarthritis.

However, according to our data beyond 10 years there is a deterioration of the result, because only 66.6 per cent of our patients are satisfied on the functional plan. Paradoxically there was a recovery in the arthritic degradation femoro-tibial medial compartment (Figure 5).

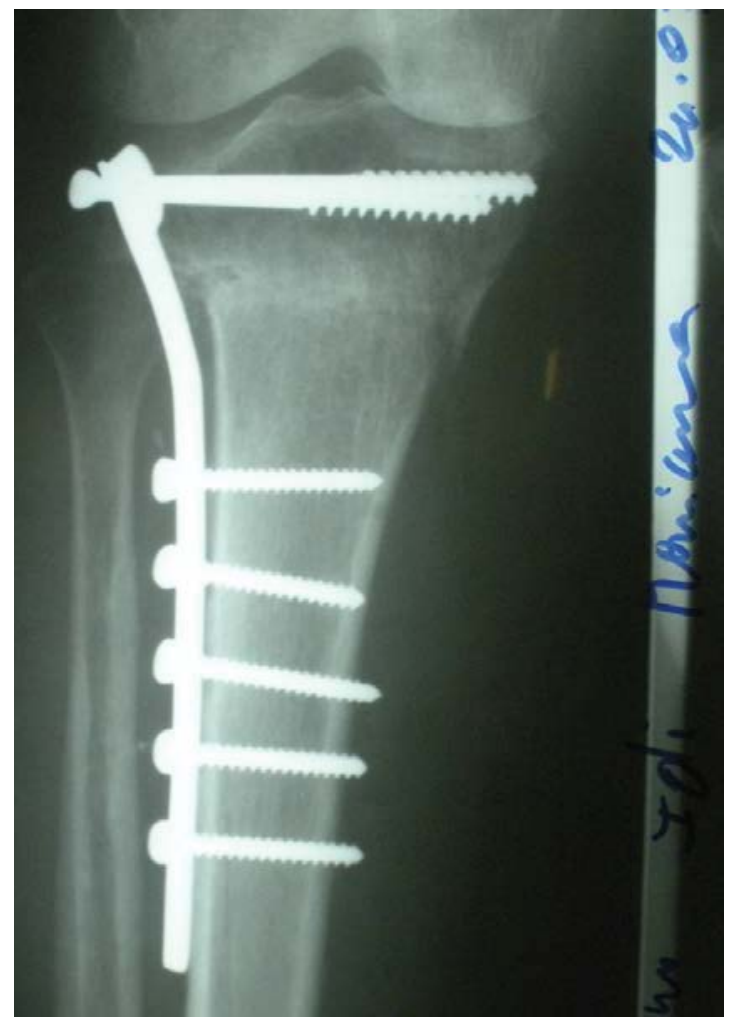

Figure 2: Hinge breakage following the osteotomy of external closure. 
Citation: Seyni SB, Amadou S, Farikou I, Zirbine AS, Wahab AM, et al. (2015) Results and Survival at Mid-term Follow-up of a Series of 15 High Tibial Lateral Closing Wedge Osteotomies in the Treatment of Unicompartimental Medial Osteoarthritis. J Trauma Treat 4: 234. doi:10.4172/2167-1222.1000234

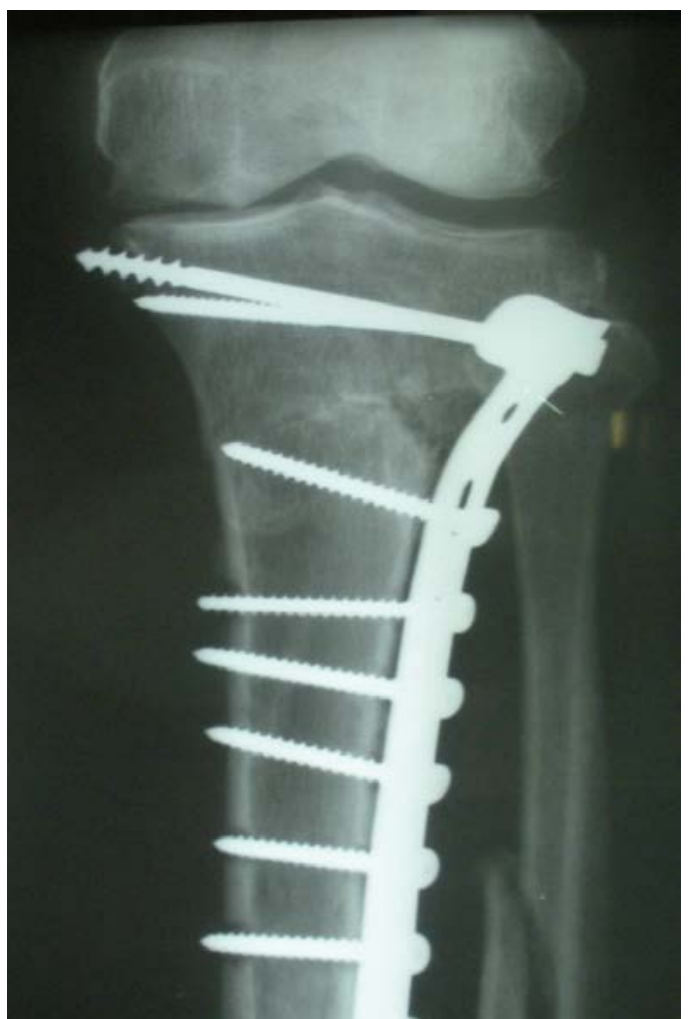

Figure 3: Type of osteotomy of closure which causes the stroke of articular refend.

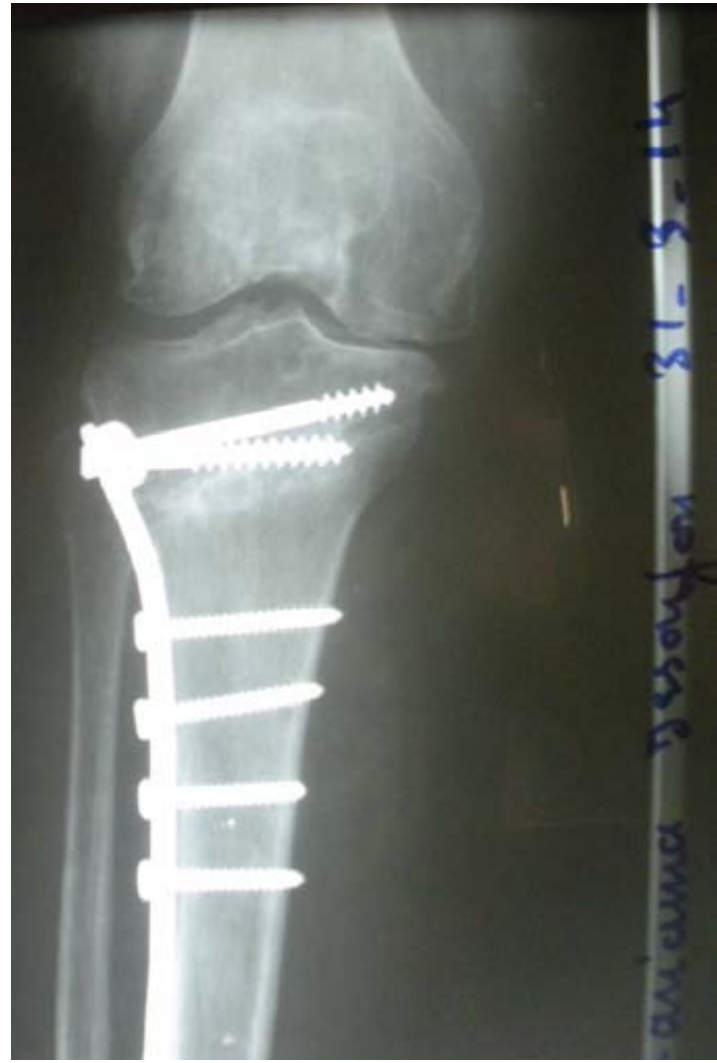

Figure 4: Consolidation of the osteotomy despite the rupture of the hinge.

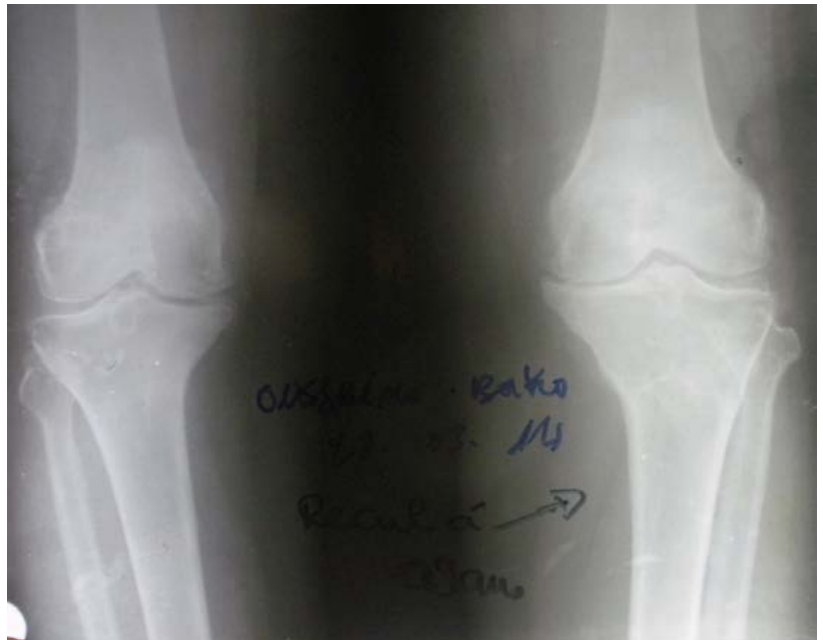

Figure 5: Loss of the correction to 12 years : degradation of the interline femoro-tibial medial of the knee operates, interline deteriorated at the same level as that of the controlateral knee non-operates.

Our survival rate at 10 years and over is lower than the rate reported by Spahn et al. [23], which was respectively $85 \%$ and $86.9 \%$ (minimum 12 years). Our low percentage of survival is explained by the fact that we have operated in majority of advanced knee osteoarthritis (stage 3 and 4 of Ahalbäck) among obese women

The analysis of some recent publications, show that both methods of osteotomy (opening and closing) gave substantially similar results.

Namely on the long-term conversion to total replacement of the knee. The conclusions of the two following authors are edifying

In their study, Duivenvoorden [24] compared the result of closing -wedge and opening-wedge high tibial osteotomy for medial compartment osteoarthritis of the knee. They found no difference in the severity of pain or in knee function between the two groups. Four complications, (9\%) occurred in the closing-wedge osteotomy group and seventeen (38\%) in the opening-wedge osteotomy group. Ten patients $(22 \%)$ in the closing -wedge group needed conversion to a total knee replacement within the six year period $(\mathrm{p}=0.05)$. Openingwedge osteotomy was associated with more complications, whereas closing -wedge osteotomy was associated with more early conversion to total knee replacement.

Soleimanpour et al. [25] in their study compared the results of treatment of genu varum using opening and closing osteotomy: they demonstrated that there is no difference between the two osteotomy methods considering patients 'clinical outcomes and both are successful in final treatment results.

\section{Conclusion}

The external wedge closing osteotomy of the proximal tibia represents among the young subject the best means of conservation of the medial femoro-tibial interline in the presence of an osteoarthritis of the latter. Well performed, the survival rate of this osteotomy at 10 ten years follow up is high. Beyond 10 years the result is degraded, imposing the requirement of prosthesis of the knee.

\section{Références}

1. Amendola A (2003) Unicompartmental osteoarthritis in the active patient: the rôle of high tibial osteotomy. Arthroscopy 19: 109-16. 
Citation: Seyni SB, Amadou S, Farikou I, Zirbine AS, Wahab AM, et al. (2015) Results and Survival at Mid-term Follow-up of a Series of 15 High Tibial Lateral Closing Wedge Osteotomies in the Treatment of Unicompartimental Medial Osteoarthritis. J Trauma Treat 4: 234. doi:10.4172/2167-1222.1000234

2. Jackson JP, Waugh W (1961) Tibial osteotomy of knee. JBJS[ Br] B: 746-751.

3. Coventry MB (1965) Osteotomy of the upper portion of the tibia for degenerative arthritis of the knee: a preliminary report. J B J S[Am] 47-A: 984-990.

4. Hernigou PH, Ovadia H, Goutallier D (1992) Mathematical modeling of the tibial osteotomy opening and correction tables. RCO 78: 258-263.

5. Bouharras M, Hoet F, Watillon M, Despontin J, Geulette R, et al. (1994) Devenir des ostéotomies tibiales valgisantes pour arthrose fémoro-tibiale interne avec un recul moyen de 8 ans. Acta Orthopoedica Belgica 60: 2 .

6. Dunoyer J, Aubriot JH, Burdin P, Delouvrier JJ, Gardes JC, et al. (1988) Gonarthrose avec genou valgum. Ann. Orthop. Ouest 20: 131-192.

7. Eti E, Kouakou HB, Daboiko JC, Ooali B, Ouattara B, et al (1998) Epidemiology and feature of knee osteoarthritis in the Ivory Coast. Rev. Rheum. (Engl. edn.) 65: $890-894$

8. Rahmi M, Ouabid A, Bekkali Y, Adnane N, Garch A, et al. (2008) Le traitement du genu varum par ostéotomie de valgisation d'addition interne par cale de ciment (à propos de 32 cas) Rev Maroc Chir Orthop Traumato 37: 21-27.

9. Naudie D, Boume RB, Rorabeck CH, Bourne TJ (1999) Survivorship of the high tibial valgus osteotomy: a 10-to 22-year followingup study. Clin Orthop Relat Res 367: 18-27.

10. Ivarsson I, Myrmerts R, Gilliquist J (1990) High tibial osteotomy for media osteoarthritis of the knee: a 5 to 7 and 11 year follow-up. JBJS [Br] 72-B: 238-244.

11. Bertin D, Karbowski A, Schwering L, Matthias HH (1998) Time dependent clinical and roentgenographical results of coven try high tibial valgisation osteotomy. Arch Orthop Trauma Surg 117: 53-57.

12. Sprenger TR, Doerzbacher JF (2003) Tibial osteotomy for the treatment of varus gonarthrosis: survival and failure analysis to twenty two years. JBJS [Am] 85-A: 469-474.

13. Insall JN, Joseph DM, Miska C (1984) High tibial osteotomy for varus gonarthrosis: a long-term follow-up study. JBJS [Am] 66-A; 1040-1048.

14. 14.Turner RS, Griffiths H, Heatley FW (1993) The incidence of deep-vein thrombosis: a veinographic study. JBJS [ Br] 75-B: 942-944.
15. Aglietti P, Buzzi R, Vena LM, Baldini A, Mondaini A (2003) High tibial valgus osteotomy for medial gonarthrosis: a 10 to 21 year study. J Kmee Surg 16 : 21-26.

16. Kessler OC, Jacob HA, Romero J (2002) Avoidance of medial cortical fracture in high tibial osteotomy: improved technique. Clin Orthop Relat Res 395: 180-185.

17. Mathews LS, Golstein SA, Malvitz TA, Katz BP, Kaufer H (1998) Proximal tibial osteotomy: factors that influence the duration of satisfactory function. $\mathrm{Cl}$ Orthop Relat Res 229: 193-200.

18. Aydogdu S, Yercan H, Saylam C, Sur H (1996) Peroneal nerve dysfunction after tibial osteotomy: an anatomical cadaver study. Acta Orthop Belg 62: 152-156.

19. Gibson MJ, Barnes MR, Allen MJ, Chan RN (1986) Weakness of foo dorsiflexion and changes in compartment pressures after tibial osteotomy. JBJS [Br] 68-B: 471-475

20. Wootton JR, Ashworth MJ, Maclaren CA (1995) Neurological complications of high tibial osteotomy: the fibular osteotomy as a causative factor a clinical and anatomical study. Am R Coll Surg Engl 77: 31-34.

21. Weingarden SI, Louis DL, Waylonis GW (1979) Electromyographic changes in post-meniscectomy patients: rôle of the pneumatic tourniquet. JAMA 241: 1248-1250.

22. Amendola A, Bonasia DE (2010) Results of high tibial osteotomy: review of the literature. Int Orthop 34: 155-160

23. Spahn G, Hofmann GO, Li M, Neubauer H, Klinger HM, et al. (2013) The impact of high tibial valgus osteotomy and unicondylar medial arthroplasty on the treatment for knee osteoarthritis: a meta-analysis. Knee Surg,Sports Traumatology, Arthroscopy 21: 96-112.

24. Duivenvoorden T, Brouwer RW, Baan A, Bos PK, Reijman M, et al (2014) Comparison of Closing-Wedge and Opening-Wedge High Tibial Osteotomy for Medial Compartment Osteoarthritis of the Knee: A Randomized Controlled Trial with a Six-Year Follow-up. J Bone Joint Surg Am 96: 1425-1432

25. Soleimanpour J, Elmi A, Jafari MA, Goldust M (2013) Comparison of genu varum treating results using open and close osteotomy. Pakistan Journal of Biological Sciences 16: 686-691. 